\title{
The polymorphism of rs2227513 can affect the expression of IL-22 and the proliferation of FLS, which leads to the progress of rheumatoid arthritis
}

\section{Type}

Research paper

\section{Keywords}

rheumatoid arthritis, IL-22, miR-101, Fibroblast-like synoviocyte, rs2227513

\begin{abstract}
Introduction

MiR-101 rs7536540 may influence the expression of miR-101 and another polymorphism, rs2227513, which in turn affects the expression of IL-22, . However, studies on the combined effect of these polymorphisms are still scarce.

Material and methods

Quantitative real-time PCR was performed to analyze the expression of miR-101 and IL-22 mRNA. ELISA and Western blot were carried out to examine the expression of IL-22 protein. MTT assay and flow cytometry were used to assess the cellular proliferation and apoptosis . Immunofluorescence was performed to measure the expression of p-STAT3. Luciferase assay was carried out to explore the inhibitory role of miR-101 in the expression of IL-22.
\end{abstract}

\section{Results}

The severity of RA was progressively increased in patients with rs7536540 GG + rs2227513 AA, rs7536540 GG + rs2227513 AG, rs7536540 CC/CG + rs2227513 AA and rs7536540 CC/CG + rs2227513 AG genotypes. The CC/CG alleles at rs 7536540 were correlated with up-regulated miR-101 expression in the serum and SF of RA patients, whereas both CC/CG alleles at rs 7536540 and AG alleles at rs2227513 were correlated with elevated expression of IL-22. Incubation of FLS with SF isolated from RA patients carrying the CC/CG alleles at rs 7536540 and AG alleles at rs2227513 remarkably increased the cell proliferation and inhibited the apoptosis of FLS. Luciferase assay demonstrated that the expression of IL-22 was notably suppressed by miR-101 in THP-1 cells.

\section{Conclusions}

Our study revealed the combined effect of polymorphisms rs7536540 and rs2227513 on the expression of IL-22 and the proliferation of FLS as well as their association with the severity of RA 
The polymorphism of rs2227513 can affect the expression of IL-22 and the proliferation of FLS, which leads to the progress of rheumatoid arthritis

\section{Abstract}

4 Background: MiR-101 rs7536540 may influence the expression of miR-101 and another polymorphism, rs2227513, which in turn affects the expression of IL-22, a gene with an essential role in the pathogenesis of RA (rheumatoid arthritis). However, studies on the combined effect of these polymorphisms are still scarce. Methods: DAS28 (ESR) and CDIA scores were measured to evaluate the severity of RA. Quantitative real-time PCR was performed to analyze the expression of miR-101 and IL-22 mRNA. ELISA and Western blot were carried out to examine the expression of IL-22 protein. MTT assay and flow cytometry were used to assess the cellular proliferation and apoptosis of FLS incubated in the SF collected from RA patients carrying differential genotypes. Immunofluorescence was performed to measure the expression of p-STAT3. Luciferase assay was carried out to explore the inhibitory role of miR-101 in the expression of IL-22. Results: The severity of RA was progressively increased in

Running title: Rs2227513 polymorphism leads to the progress of RA by affecting the expression 
Rheumatoid arthritis (RA) is a systemic, inflammatory autoimmune disease which is commonly considered as joint disease despite that multiple additional organ systems such as pulmonary, cardiovascular, ocular, and cutaneous systems are known to be involved [1]. RA often triggers evident joint damages, inflammatory arthritis (IA), and bone loss [2, 3]. However, despite all these symptoms, the presence of IA is the hallmark of clinical diagnosis of RA. The diagnosis criteria is compromised as a series of joint examination and imaging, testing of RA-related autoantibodies, systemic inflammation, and the duration of joint symptoms $[1,4]$.

Non-coding RNAs (ncRNAs) are primarily separated into 2 sub-types based upon their lengths: long non-coding RNAs (IncRNAs) as well as microRNAs (miRNAs). LncRNAs are specified as a type of transcripts with no protein coding ability that are greater than 200 nucleotides in length. IncRNAs are keys in many biological processes, including transcription as well as epigenetic regulation [6]. MiRNAs are short, single-stranded RNAs participating in the control of different biological activities, including cell growth, apoptosis, inflammation, as well as tumorigenesis [7].

Previous research revealed that $\mathrm{rs} 7536540$ on miR-101-1 can bind to various gene regulators. More lately, the ATP synthase, $\mathrm{H}+$ transporting, mitochondrial F1 complex, beta polypeptide (ATP5B) was revealed to be inhibited by miR-101 [8]. Given that ATP5B expression is selectively suppressed in human tumors, it is suspected that rs 7536540 induces miR-101 overexpression to reduce ATP5B expression while indirectly influencing the progression of HBV-related illness [9].

Interleukin (IL)-22 is an IL-10 family member of cytokines, and it has also been demonstrated that IL-22 expression is dysregulated in RA patients and the aberrant IL-22 is significantly correlated with the onset of RA [10]. Accordingly, in a previous study, IL-22 was suggested to exhibit therapeutic effect in the treatment of RA from the aspect of immune mechanisms rather than the aspect of genetic susceptibility [10]. A vast array of immune cells, including T cells, natural killer (NK) cells, dendritic cells, monocytes, as well as lymphoid cells, can generate IL-22 [11-13]. However, the key resource of IL-22 is activated T cells, especially Th17, Th1, as well as Th22 cells [12]. Ikeuchi and his colleagues have examined and showed that IL-22 can 
57 boost phospho-ERK1/2 expression in synovial fibroblasts [14]. Taking into consideration that STAT3-mediated signaling is an often seen route shared by the members of the IL-10 family, the STAT3 pathway is indeed activated by rhlL-22 through boosting phospho-STAT3 expression, which is due to the fact that AG490 inhibits the expansion of fibroblast-like synoviocytes (FLS) [15]. Similarly, STAT3 phosphorylation is a vital pathway moderating the effect of IL-22 on FLS

81 the protocol of this study. 
The expression of miR-101 and IL-22 mRNA in the serum samples collected from the subjects was measured using real time PCR. In brief, the samples were treated with the assay kits of mirVana (Ambion, Thermo Fisher Scientific, Waltham, MA), Trizol Reagent (Invitrogen, Carlsbad, CA), and miRNeasy (Qiagen, Redwood City, CA) following the instructions of the manufacturers to collect their total RNA contents. In the next step, the RNA extract was rehydrated on a column and then centrifuged at $4000 \mathrm{~g}$ and $4^{\circ} \mathrm{C}$ to accumulate the RNA eluate. Finally, all extracted RNA was precipitated first and then resuspended in nuclease free water (Invitrogen, Carlsbad, CA) for subsequent analysis.

During real time PCR, the extracted RNA was first reversely transcribed into CDNA by using a TaqMan miRNA Reverse Transcription Assay kit following the instructions of the manufacturer, followed by real time RT-PCR carried out using a SYBR Green real time PCR kit (Thermo Fisher Scientific, Waltham, MA) following the instructions of the manufacturer.

\section{Cell culture and transfection}

THP-1 cells were purchased from European Collection of Cell Cultures (Salisbury, United Kingdom) and incubated at $37^{\circ} \mathrm{C}$ in a humidified carbon dioxide incubator. The culture medium was Dulbecco's Modified Eagle's Medium (DMEM, Sigma Aldrich, St. Louis, MO) supplemented with $10 \%$ fetal calf serum (Gibco, Thermo Fisher Scientific, Waltham, MA) as well as $1 \%$ of penicillin and streptomycin (Invitrogen, Carlsbad, CA). Prior to the transfection experiments, the cells reached logarithmic growth and were divided into 3 groups, i.e., 1. NC; 2. miR-101 mimics; and 3. IL-22 siRNA. The cells were transfected with respective oligonucleotides (Saigon, Shanghai, China) for $48 \mathrm{~h}$ by using Lipofectamine 3000 (Invitrogen, Carlsbad, CA) following the instructions of the manufacturer. Then, the cells were harvested to measure the expression of target genes.

\section{Vector construction, mutagenesis and luciferase assay}

We carried out a binding site screening to search potential target genes of miR-101 and found that the 3' UTR of IL-22 contained a potential miR-101 binding site. Then, the 3' UTR of IL-22 containing the miR-101 binding site was inserted into a pcDNA3.1 vector downstream of the firefly luciferase reporter gene to generate the wild type vector of IL-22 3' UTR. At the same 
111 time, a Quick Change mutagenesis kit (Stratagene, San Diego, CA) was used to generate a site 112 directed mutation in the 3' UTR of IL-22, which was also inserted into a pcDNA3.1 vector to 113 create the mutant type vector of IL-22 3' UTR. In the next step, the wild type or mutant type 114 vector of IL-22 3' UTR was co-transfected with miR-101 mimics into THP-1 cells, which were 115 pre-seeded in 96 well tissue culture plates at 50000 cells/well at $24 \mathrm{~h}$ prior to transfection. After $11648 \mathrm{~h}$ of transfection, the luciferase activity of transfected cells was assayed using a Bright Glo 117 luciferase assay kit (Promega, Madison, WI) following the instructions of the manufacturer. The 118 reading of luciferase activity was carried out on a TD 20/20 luminometer (Turner Styles, 119 Sunnyvale, CA).

\section{Cell proliferation assay}

121 THP-1 cells were treated and plated into 96 well cell culture plates at 50000 cells/well in $100 \mu \mathrm{L}$ 122 of medium. The proliferation status of the cells was assayed by using an MTT assay kit (Sigma 123 Aldrich, St. Louis, MO) following the instructions of the manufacturer. The optical density values of various wells were measured at $450 \mathrm{~nm}$ on a plate reader.

\section{Western blot analysis}

Western blot was carried out using a conventional method. In brief, the collected clinical and cell culture samples were homogenated in a TNEC lysis buffer $(\mathrm{pH}$ 8) containing $1.5 \mathrm{mM}$ of Tris$\mathrm{HCl}, 2 \mathrm{mM}$ of $\mathrm{NaCl}, 3 \%$ of IGEPAL CA-630, $4 \mathrm{mM}$ of EDTA and a cocktail of protease inhibitors (Roche, Switzerland). The protein content in the homogenate was assayed by making use of a BCA assay kit (Pierce, Waltham, MA) following the instructions of the kit manufacturer. About $40 \mu \mathrm{g}$ of protein sample from each specimen were denatured by using a LDS buffer containing a NuPAGE denaturing agent (both from Invitrogen, Carlsbad, CA) that was warmed up to $72{ }^{\circ} \mathrm{C}$ in and incubated for 10 minutes. In the next step, the proteins were resolved on a $8 \%$ TrisAcetate gel (Invitrogen, Carlsbad, CA), moved to a nitrocellulose membrane, blocked in a TBST buffer containing 5\% non-fat milk, and incubated consecutively with primary anti- IL-22 136 antibodies and appropriate secondary antibodies (Santa Cruz Biotechnology, San Diego, CA) 137 before the relative protein expression of IL-22 in each sample was evaluated by utilizing an AlphaEaseFC system (Alpha Innotech, San Leandro, CA). 
The apoptosis of cells was evaluated using flow cytometry. In brief, THP-1 cells were collected at $48 \mathrm{~h}$ post transfection when the cell confluency reached about $80 \%$ ). Then, $0.4 \times 10^{6}$ cells were obtained for each sample and treated with an Annexin V Apoptosis Assay Kit (Thermo 143 Fisher Scientific, Waltham, MA) following the instructions of the kit manufacturer. The assay 144 values were read on a FACS Caliber flow cytometer using Summit 3.1 software (Beckman 145 Coulter, San Jose, CA).

\section{Immunofluorescence}

147 The expression of p-STAT3 in samples was determined using an immunofluorescence assay. In 148 brief, the cells were seeded on glass cover slides and then fixed by PBS containing $4 \%(v / v)$ paraformaldehyde. After being permeabilized using PBS with 0.5\% (v/v) Triton X-100 for 15 minutes, the samples were blocked by using PBS with 5\% (v/v) FBS for $1 \mathrm{~h}$. In the next step, the

151 cells were incubated with primary anti-p-STAT3 antibody and corresponding AF-488-tagged Ig G 152 secondary antibody (Molecular Probes, Carlsbad, CA) following the instructions of the 153 manufacturer. The positive expression of p-STAT3 in samples was analyzed underneath a 154 fluorescence microscope (Axio Imager A1, ZEISS, Germany).

\section{ELISA}

156 The expression of IL-22 in SF samples was measured by using an ELISA assay kit (BD, Franklin 157 Lakes, NJ) following the instructions of the kit manufacturer, and the results were read at an 158 excitation wavelength of 450 and an emission wavelength of $620 \mathrm{~nm}$ on a plate reader (BioRad 159 Laboratories, Richmond, CA).

\section{Statistical analysis}

161 All statistical evaluations were done by making use of GraphPad Prism 6.0 software (GraphPad, 162 San Diego, CA). The experimental values were expressed by mean \pm SEM. Inter group 163 comparisons were done using Student's t-test (two trailed, non-parametric) and the statistical 164 difference was determined by $\mathrm{P} \leq 0.05$. 


\section{Results}

Demographic and clinicopathological characteristics of the participants recruited in the present study

We have recruited a total of 84 RA (Rheumatoid Arthritis) patients in this study and partitioned them into 4 groups according to their genotypes of rs7536540 and rs2227513: 1. miR-101 rs7536540 GG + IL-22 rs2227513 AA (N=18); 2.miR-101 rs7536540 GG + IL-22 rs2227513 AG ( $N=22)$; 3. miR-101 rs7536540 CC/CG + IL-22 rs2227513 AA ( $=24)$ and 4. miR-101 rs7536540 $\mathrm{CC} / \mathrm{CG}+\mathrm{IL}-22$ rs2227513 AG (N=20). The demographic and clinicopathological features of the subjects were summarized in Table 1 . The differences among the four groups were compared using Student's t test and no obvious were seen.

The severity of RA was elevated in patients with CC/CG alleles at rs7536540 and AG alleles at rs2227513.

DAS28 (ESR) was measured in each group of patients to evaluate the severity of RA. The DAS28 (ESR) was the smallest in patients with GG alleles at rs 7536540 and AA alleles at rs2227513, but the largest in patients with CC/CG alleles at rs7536540 and AG alleles at rs2227513. The DAS28 (ESR) was at a moderate level in patients with GG alleles at rs7536540 + AA alleles at rs2227513 and CC/CG genotypes at rs7536540 + AA alleles at rs2227513 (Fig.1A). Furthermore, the CDIA scores in the patients showed the same trends as those of DAS28 (ESR) in different groups (Fig.1B). These results indicated that CC/CG at rs7536540 and AG at rs2227513 significantly increased the severity of RA.

\section{The expression of miR-101 and IL-22 was elevated in patients with CC/CG alleles at rs7536540} and AG alleles at rs2227513.

Next, the serum and SF (synovial fluid) of RA patients were collected and subjected to measurement of miR-101 and IL-22 expression. No obvious difference was observed for the expression of miR-101 between patients carrying AA or AG alleles at rs2227513, whereas the serum and SF expression of miR-101 in patients with CC/CG alleles at rs7536540 was significantly higher than that in patients with GG alleles at rs7536540 (Fig.2A and 3A). However, 
192 the expression of IL-22 in the SF progressively increased in patients with rs7536540 GG + rs2227513 AA, rs7536540 GG + rs2227513 AG, rs7536540 CC/CG + rs2227513 AA and rs7536540 CC/CG + rs2227513 AG (Fig.2B and 3B). These results demonstrated that miR-101 rs7536540 effectively altered the expression of miR-101, and both miR-101 rs7536540 and IL-22 rs2227513 altered the expression of IL-22 in the serum and SF of RA patients.

CC/CG alleles at rs7536540 and AG alleles at rs2227513 were correlated with elevated 198 proliferation and decreased apoptosis of fibroblast-like synoviocytes incubated with SF isolated from RA patients.

Fibroblast-like synoviocytes were incubated for 48 hours with SF collected from RA patients carrying differential genotypes, followed by MTT and flow cytometry analysis to evaluate their proliferation and apoptosis. The proliferation showed an obviously elevating trend when fibroblast-like synoviocytes were incubated with SF from RA patients with genotypes of rs7536540 GG + rs2227513 AA, rs7536540 GG + rs2227513 AG, rs7536540 CC/CG + rs2227513 205 AA, and rs7536540 CC/CG + rs2227513 AG (Fig.4). Besides, the apoptosis of fibroblast-like synoviocytes was gradually suppressed when they were incubated with SF from RA patients carrying genotypes of rs7536540 GG + rs2227513 AA, rs7536540 GG + rs2227513 AG, rs7536540 CC/CG + rs2227513 AA, and rs7536540 CC/CG + rs2227513 AG (Fig.5). Moreover, were incubated with SF collected from RA patients carrying genotypes of rs7536540 GG + rs2227513 AA, rs7536540 GG + rs2227513 AG, rs7536540 CC/CG + rs2227513 AA, and rs7536540 CC/CG + rs2227513 AG (Fig.6).

MiR-101 mimics and IL-22 siRNA significantly suppressed the expression of IL-22 in THP-1 cells.

215 Transfection of miR-101 mimics and IL-22 siRNA was performed to evaluate their effect on IL-22 216 expression in THP-1 cells. The expression of IL-22 in THP-1 cells transfected with miR-101 217 mimics and IL-22 siRNA was remarkably suppressed. 
A screening of binding sites of miR-101 found that the 3' UTR of IL-22 was a potential target of miR-101 (Fig.8A). Next, luciferase vectors containing wild type and mutant 3' UTRs of IL-22 were established and co-transfected into THP-1 cells with miR-101. The luciferase activity of wild type IL-22 vector was effectively suppressed by miR-101, whereas the luciferase activity of mutant IL-22 remained unchanged (Fig.8B).

\section{Discussion}

It has been previously reported that rs7536540 may influence the expression of miR-101 while rs2227513 may affect the expression of IL-22. In this study, we hypothesized that the genotypes of these two polymorphisms may exhibit a combined effect upon the severity of RA by influencing the expression of IL-22 and the proliferation of FLS. Accordingly, our study found that patients with rs7536540 genotype as CC/CG and rs2227513 genotype as AG were suffering from higher severity of RA with elevated expression of miR-101 and IL-22. And for fibroblastlike synoviocytes isolated from these patients, their proliferation was promoted while the apoptosis was suppressed. Accordingly, we studied the underlying molecular mechanism and found that miR-101 negative regulated the expression of IL-22 through binding to the 3'UTR of IL-22 mRNA. Therefore, our study not only validated the correlation between polymorphism genotypes and RA severity, but also established the underlying molecular mechanisms.

RA is a persistent and inflammatory condition that assaults synovial joint [19]. FLS are cells first impacted by the development of arthritis [20]. It was shown that IL-22 as well as its receptors in synovial cells and FLS are overexpressed along with increased levels of Th17 cells in the joint of individuals with RA [14, 21, 22]. Likewise, high IL-22 expression in the synovial fluid of RA subjects is associated with disease development $[23,24]$. FLS show a main function in RA subjects with synovial hyperplasia [20]. The level of FLS is evidently raised in the synovial cells of RA subjects, along with damaged cartilage materials as well as activation of proinflammatory cytokines, Matrix Metallaproteinases (MMPs), and chemokines [20, 25].

The function of IL-22 in antiviral protection remains debatable. Located on the IL-22 gene, the A/G genotype of rs2227513 is shown to raise the danger of HIV infection, no matter whether 
246 the subject is HIV seronegative or seropositive, indicating a pathogenic effect of IL-22 on the 247 infection of HIV $[26,27]$.

248 Rs2227513 is situated on an intron in IL-22. It is known that introns can work as crucial gene 249 regulatory elements exerting different impacts, including RNA modification [26, 27]. A statistical 250 correlation was seen between genotype distribution and rs2227513 alleles in kidney diseases, 251 indicating a close association between the genotypes of rs2227513 and the risk of HIV infection 252 amongst females [28].

253 Current research shows that miR-101-3p level is evidently lowered amongst synovium as well as 254 FLS collected from AIA animals. Transfection with miR-101-3p mimic evidently minimized the 255 expression of CUL4B, while miR-101-3p inhibitor evidently upregulated the level of CUL4B 256 amongst AIA FLS. Previous researches have revealed that miR-101 is closely associated with 257 tumor malignancy. The irregular expression of miR-101 was verified in several studies 2, 3. 258 Research has also revealed that miR-101 is linked to tumor metastasis. As an example, subduing 259 miR-101 expression enhanced the metastasis of cancer cells 4 . It was also revealed that miR101 silencing could prevent cartilage destruction [29]. Dual specificity phosphatase 1 (DUSP1), or MKP-1, could undermine the activation of MAPK signaling in arthritis [30]. Wei et al. (2015) 262 confirmed that DUSP1 acts as a target of miR-101. In summary, it was hypothesized that miR263101 overexpression may target DUSP1 to enhance cartilage deterioration in RA [31]. In this 264 study, we recruited RA patients and sub-grouped them into 4 groups according to their 265 genotypes of rs7536540 and rs2227513. Accordingly, the severity of RA was remarkable 266 elevated in patients carrying the genotypes of rs7536540 CC/CG + rs2227513 AG compared 267 with other patient groups. CC/CG at rs7536540 was correlated with up-regulated miR-101 268 expression, while CC/CG at rs7536540 and AG at rs2227513 were correlated with up-regulated 269 IL-22 expression in the serum and SF of RA patients.

270 IL-22 is generated by NK cells, activated T cells, as well as lymphoid cells [32]. The secretion of 271 IL-22 as well as CCL2 by eESCs promoted the recruitment of macrophages to advance 272 endometriosis. Similarly, the co-culture of NK cells with eESCs promoted the secretion of IL-22 273 and CCL2 to promote the recruitment of macrophages. The IL-10 family includes cytokines such 
274 as IL-26, IL-10, IL-22, IL-19, IL-24, as well as IL-20, which are generated by various immune cells 275 to play roles as effectors in the immune system. IL-22 can trigger numerous immune responses. 276 For example, IL-22 promotes the immune responses in the liver by activating different anti277 microbial pathways, including HPX, C3, as well as hepcidin. In this study, we incubated FLS for 27848 hours in SF isolated from RA patients and performed MTT assay and flow cytometry to 279 evaluate the proliferation and apoptosis of FLS. SF isolated from patients carrying the 280 genotypes of rs7536540 GG + rs2227513 AA, rs7536540 GG + rs2227513 AG, rs7536540 CC/CG + rs2227513 AA, and rs7536540 CC/CG + rs2227513 AG gradually increased the proliferation 282 and decreased the apoptosis of FLS.

283 Formerly, Ikeuchi et al. revealed that IL-22 is likewise synthesized as a type of proinflammatory 284 cytokine in macrophages as well as synovial fibroblasts to enhance inflammatory reactions via 285 the IL-22 receptor 1 located on the surface of FLS in RA subjects [14]. The results of MTT assays 286 showed that the supernatants collected from the culture of NKp44pNK cells enhanced the 287 expansion of FLS, while IL-22 antagonist could suppress the effects of the above supernatants.

\section{Conclusion}

289 In summary, the findings of this study demonstrated that miR-101 rs7536540 may influence the 290 expression of miR-101 and another polymorphism, rs2227513, to affect the expression of IL291 22. In this study, we investigated the combined effect of these two polymorphisms on the 292 expression of IL-22 and the proliferation of FLS as well as their association with the severity of 293 RA.

294 Conflict of interest

295 None

296 Funding information

297 This study was sponsored by Health and Family Planning Commission of Jiangxi Province 298 (20161020).

299 Figure legends 
301 Demographic and clinicopathological parameters of recruited subjects

$302 \quad$ Fig. 1

303 CC/CG alleles at rs7536540 and AG alleles at rs2227513 were correlated with elevated severity 304 of RA.

305 A: DAS28 (ESR) was progressively increased in RA patients carrying the genotypes of rs7536540 306 GG + rs2227513 AA, rs7536540 GG + rs2227513 AG, rs7536540 CC/CG + rs2227513 AA, and 307 rs7536540 CC/CG + rs2227513 AG.

308 B: CDIA score was progressively increased in RA patients carrying the genotypes of rs7536540 309 GG + rs2227513 AA, rs7536540 GG + rs2227513 AG, rs7536540 CC/CG + rs2227513 AA, and $310 \quad r s 7536540 \mathrm{CC} / \mathrm{CG}+\mathrm{rs} 2227513$ AG.

$311 \quad$ Fig. 2

312 CC/CG alleles at rs7536540 and AG alleles at rs2227513 were correlated with enhanced 313 expression of miR-101 and IL-11 in the serum of RA patients.

314 A: The expression of miR-101 was significantly elevated in the serum of RA patients with CC/CG 315 alleles at rs7536540.

316 B: The expression of IL-22 was gradually enhanced in the serum of RA patients carrying the 317 genotypes of rs7536540 GG + rs2227513 AA, rs7536540 GG + rs2227513 AG, rs7536540 CC/CG $318+$ rs2227513 AA, and rs7536540 CC/CG + rs2227513 AG.

$319 \quad$ Fig. 3

320 CC/CG alleles at rs7536540 and AG alleles at rs2227513 were correlated with enhanced 321 expression of miR-101 and IL-11 in the SF of RA patients.

322 A: The expression of miR-101 was significantly elevated in the SF of RA patients with CC/CG 323 alleles at rs7536540. 
324 B: The expression of IL-22 was gradually enhanced in the SF of RA patients carrying the 325 genotypes of rs7536540 GG + rs2227513 AA, rs7536540 GG + rs2227513 AG, rs7536540 CC/CG $326+$ rs2227513 AA, and rs7536540 CC/CG + rs2227513 AG.

$327 \quad$ Fig. 4

328 The viability of FLS was gradually elevated when incubated with SF isolated from RA patients 329 carrying the genotypes of rs7536540 GG + rs2227513 AA, rs7536540 GG + rs2227513 AG, $330 \mathrm{rs} 7536540 \mathrm{CC} / \mathrm{CG}+\mathrm{rs} 2227513 \mathrm{AA}$, and rs7536540 CC/CG + rs2227513 AG.

$331 \quad$ Fig. 5

332 The apoptosis of FLS was progressively decreased when incubated with SF isolated from RA 333 patients carrying the genotypes of rs7536540 GG + rs2227513 AA, rs7536540 GG + rs2227513 334 AG, rs7536540 CC/CG + rs2227513 AA, and rs7536540 CC/CG + rs2227513 AG.

335 Fig. 6

336 The expression of p-STAT3 was gradually elevated when incubated with SF isolated from RA 337 patients carrying the genotypes of rs7536540 GG + rs2227513 AA, rs7536540 GG + rs2227513 338 AG, rs7536540 CC/CG + rs2227513 AA, and rs7536540 CC/CG + rs2227513 AG.

$339 \quad$ Fig. 7

340 The expression of IL-22 in THP-1 cells was inhibited by miR-101 mimics and IL-22 siRNA.

341 A: The expression of IL-22 mRNA was remarkably suppressed by miR-101 mimics and IL-22 342 siRNA (* P value $<0.05$, vs. NC group).

343 B: The expression of IL-22 protein was remarkably suppressed by miR-101 mimics and IL-22 344 siRNA ( $*$ P value $<0.05$, vs. NC group).

$345 \quad$ Fig. 8

346 MiR-101 inhibited the expression of IL-22 through binding to its 3' UTR.

347 A: Sequence analysis indicated a potential binding site of miR-101 on IL-22. 
348 B: The luciferase activity of wild type IL-22 was effectively repressed by miR-101 (* P value < 3490.05, vs. control group).

\section{References}

3511 Deane KD, Holers VM: The Natural History of Rheumatoid Arthritis. Clin Ther 2019; 352 41(7):1256-1269.

3532 Elbinoune I, Amine B, Wabi M, Rkain H, Aktaou S, Hajjaj-Hassouni N: Rheumatoid 354 shoulder assessed by ultrasonography: prevalence of abnormalities and associated 355 factors. Pan Afr Med J 2016;24:235.

3563 Rittmeister M, Kerschbaumer F: Grammont reverse total shoulder arthroplasty in 357 patients with rheumatoid arthritis and nonreconstructible rotator cuff lesions. J 358 Shoulder Elbow Surg 2001;10:17-22.

3594 Balbaloglu O, Ozcan S: Is pentraxin 3 level an effective biomarker in disease activity in 360 patients with rheumatoid arthritis?. Arch Med Sci 2020;16(1):81-86.

3615 Brosius J: Waste not, want not--transcript excess in multicellular eukaryotes. Trends $362 \quad$ Genet 2005;21:287-288.

3636 Mercer TR, Dinger ME, Mattick JS: Long non-coding RNAs: insights into functions. Nat $364 \quad$ Rev Genet 2009;10:155-159.

3657 Paladini L, Fabris L, Bottai G, Raschioni C, Calin GA, Santarpia L: Targeting microRNAs as 366 key modulators of tumor immune response. J Exp Clin Cancer Res 2016;35:103.

3678 Zheng SQ, Li YX, Zhang Y, Li X, Tang H: MiR-101 regulates HSV-1 replication by targeting 368 ATP5B. Antiviral Res 2011;89:219-226.

3699 Willers IM, Isidoro A, Ortega AD, Fernandez PL, Cuezva JM: Selective inhibition of beta370 F1-ATPase mRNA translation in human tumours. Biochem J 2010;426:319-326.

37110 Xie Q, Huang C, Li J: Interleukin-22 and rheumatoid arthritis: emerging role in 372 pathogenesis and therapy. Autoimmunity 2015;48(2):69-72.

37311 Dezso K, Jelnes P, Laszlo V, Baghy K, Bodor C, Paku S, Tygstrup N, Bisgaard HC, Nagy P: 374 Thy-1 is expressed in hepatic myofibroblasts and not oval cells in stem cell-mediated 375 liver regeneration. Am J Pathol 2007;171:1529-1537. 
37612 Chen E, Cen Y, Lu D, Luo W, Jiang H: IL-22 inactivates hepatic stellate cells via 377 downregulation of the TGF-beta1/Notch signaling pathway. Mol Med Rep $378 \quad 2018 ; 17: 5449-5453$.

37913 Sonnenberg GF, Fouser LA, Artis D: Border patrol: regulation of immunity, inflammation 380 and tissue homeostasis at barrier surfaces by IL-22. Nat Immunol 2011;12:383-390.

38114 Ikeuchi H, Kuroiwa T, Hiramatsu N, Kaneko Y, Hiromura K, Ueki K, Nojima Y: Expression 382 of interleukin-22 in rheumatoid arthritis: potential role as a proinflammatory cytokine. 383 Arthritis Rheum 2005;52:1037-1046.

38415 Yang X, Zheng SG: Interleukin-22: a likely target for treatment of autoimmune diseases. 385

38616 Shaker O, Alhelf M, Morcos G, Elsharkawy A: miRNA-101-1 and miRNA-221 expressions Autoimmun Rev 2014;13:615-620. and their polymorphisms as biomarkers for early diagnosis of hepatocellular carcinoma. Infect Genet Evol 2017;51:173-181.

17 Pratedrat P, Sopipong W, Makkoch J, Praianantathavorn K, Chuaypen N, Tangkijvanich P, 390

18 Wang R, Zeng YL, Qin HM, Lu YL, Huang HT, Lei M, Tan T, Huang YY, Luo HC, Lan Y, Wei 394 Payungporn S: Single Nucleotide Polymorphisms in miR-149 (rs2292832) and miR-101-1 (rs7536540) Are Not Associated with Hepatocellular Carcinoma in Thai Patients with Hepatitis B Virus Infection. Asian Pac J Cancer Prev 2015;16:6457-6461.

39719 Firestein GS: Evolving concepts of rheumatoid arthritis. Nature 2003;423:356-361.

39820 Bartok B, Firestein GS: Fibroblast-like synoviocytes: key effector cells in rheumatoid $399 \quad$ arthritis. Immunol Rev 2010;233:233-255.

40021 Pene J, Chevalier S, Preisser L, Venereau E, Guilleux MH, Ghannam S, Moles JP, Danger Y, 401 Ravon E, Lesaux S, Yssel H, Gascan H: Chronically inflamed human tissues are infiltrated 402 by highly differentiated Th17 lymphocytes. J Immunol 2008;180:7423-7430. 
22 Shen H, Goodall JC, Hill Gaston JS: Frequency and phenotype of peripheral blood Th17 cells in ankylosing spondylitis and rheumatoid arthritis. Arthritis Rheum 2009;60:16471656.

23 Cascao R, Moura RA, Perpetuo I, Canhao H, Vieira-Sousa E, Mourao AF, Rodrigues AM,

24 Leipe J, Schramm MA, Grunke M, Baeuerle M, Dechant C, Nigg AP, Witt MN, Vielhauer V, radiographic progression in rheumatoid arthritis. Ann Rheum Dis 2011;70:1453-1457.

Muller-Ladner U, Kriegsmann J, Franklin BN, Matsumoto S, Geiler T, Gay RE, Gay S: Synovial fibroblasts of patients with rheumatoid arthritis attach to and invade normal human cartilage when engrafted into SCID mice. Am J Pathol 1996;149:1607-1615. Jacquier A: Group II introns: elaborate ribozymes. Biochimie 1996;78:474-487. Qaddourah RH, Magdoud K, Saldanha FL, Mahmood N, Mustafa FE, Mahjoub T, Almawi WY: IL-10 gene promoter and intron polymorphisms and changes in IL-10 secretion in women with idiopathic recurrent miscarriage. Hum Reprod 2014;29:1025-1034.

Hu J, Li Y, Chen L, Yang Z, Zhao G, Wang Y, Cheng J, Zhao J, Peng Y: Impact of IL-22 gene polymorphism on human immunodeficiency virus infection in Han Chinese patients. J Microbiol Immunol Infect 2016;49:872-878. Prevents Cartilage Degradation by Regulating Extracellular Matrix-related Genes in a Rat Model of Osteoarthritis. Mol Ther 2015;23:1331-1340.

30 Vattakuzhi Y, Abraham SM, Freidin A, Clark AR, Horwood NJ: Dual-specificity phosphatase 1-null mice exhibit spontaneous osteolytic disease and enhanced inflammatory osteolysis in experimental arthritis. Arthritis Rheum 2012;64:2201-2210.

31 Wei X, Tang C, Lu X, Liu R, Zhou M, He D, Zheng D, Sun C, Wu Z: MiR-101 targets DUSP1 to regulate the TGF-beta secretion in sorafenib inhibits macrophage-induced growth of hepatocarcinoma. Oncotarget 2015;6:18389-18405. 
43232 Nikoopour E, Bellemore SM, Singh B: IL-22, cell regeneration and autoimmunity. Cytokine 2015;74:35-42. 


\begin{tabular}{lccccc}
\hline \multicolumn{1}{c}{ Characteristics } & $\begin{array}{c}\text { miR-101 rs7536540 GG + } \\
\text { IL-22 rs2227513 AA (N=18) }\end{array}$ & $\begin{array}{c}\text { miR-101 rs7536540 GG }+ \\
\text { IL-22 rs2227513 AG (N=22) }\end{array}$ & $\begin{array}{c}\text { miR-101 rs7536540 CC/CG } \\
+ \text { IL-22 rs2227513 AA } \\
\text { (N=24) }\end{array}$ & $\begin{array}{c}\text { miR-101 rs7536540 CC/CG } \\
+ \text { IL-22 rs2227513 AG } \\
\text { (N=20) }\end{array}$ & $\begin{array}{c}\boldsymbol{P} \text { value } \\
\text { Age (years) at } \\
\text { enrolment }\end{array}$ \\
Female sex, $\mathrm{n}(\%)$ & $42.8 \pm 5.4$ & $43.3 \pm 5.7$ & $40.3 \pm 3.6$ & $41.7 \pm 5.7$ & 0.284 \\
Disease duration, years & $12(66.7)$ & $15(68.2)$ & $18(75.0)$ & $15(75.0)$ & 0.594 \\
CRP, mg/L & $6.0 \pm 1.1$ & $6.4 \pm 0.9$ & $6.0 \pm 1.3$ & $6.4 \pm 0.8$ & 0.698 \\
ESR, mm/h & $25.4 \pm 5.7$ & $25.0 \pm 6.7$ & $25.3 \pm 5.7$ & $25.4 \pm 5.7$ & 0.753 \\
\hline Table 1. Demographic and clinicopathological parameters of recruited subjects & $51.4 \pm 6.2$ & $49.2 \pm 7.9$ & $50.6 \pm 5.3$ & 0.374 \\
\hline
\end{tabular}

Table 1. Demographic and clinicopathological parameters of recruited subjects 

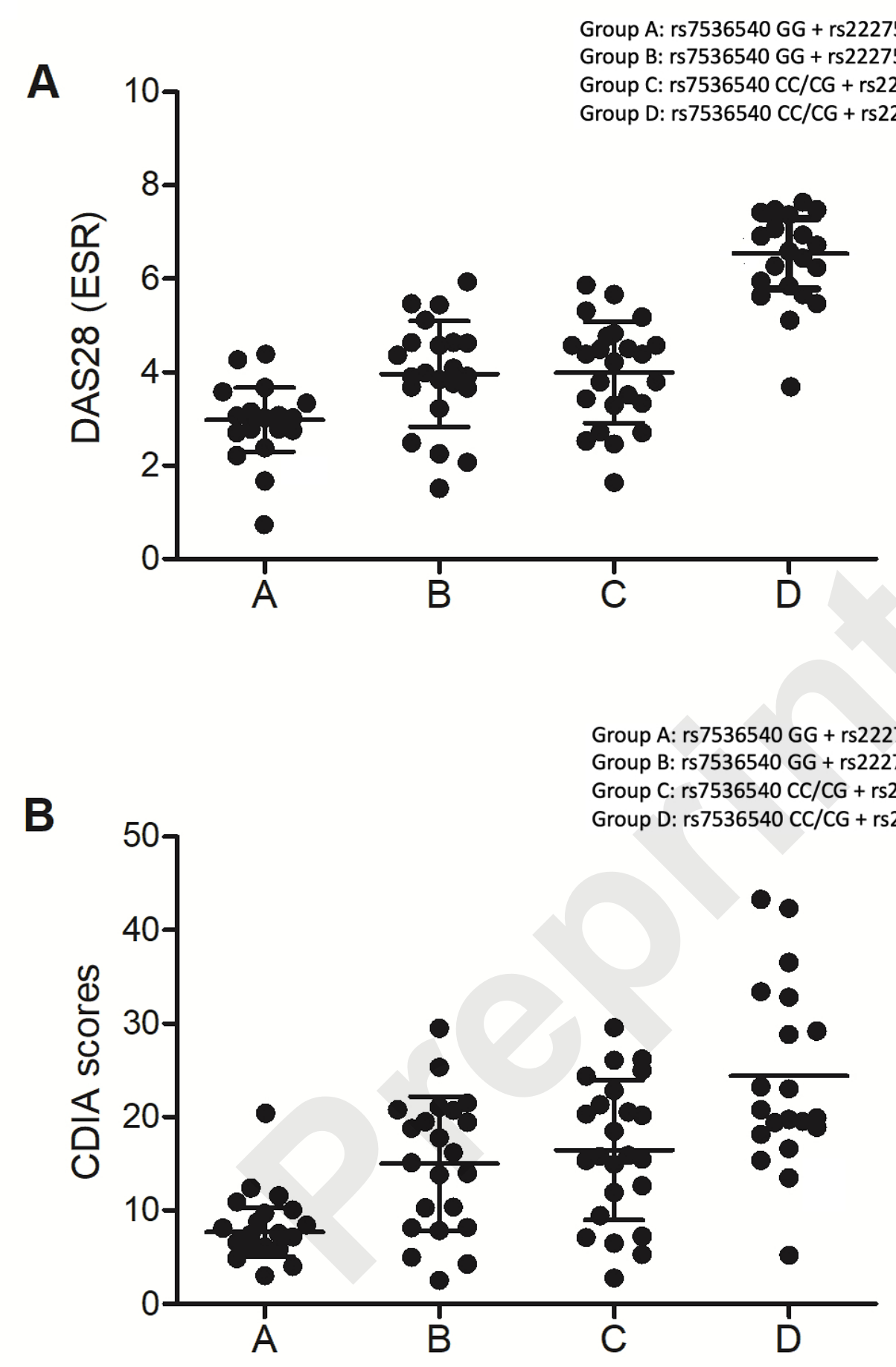

Fig. 1

CC/CG alleles at rs7536540 and AG alleles at rs2227513 were correlated with elevated severity of RA.

A: DAS28 (ESR) was progressively increased in RA patients carrying the genotypes of rs7536540 GG + rs2227513 AA, rs7536540 GG + rs2227513 AG, rs7536540 CC/CG + rs2227513 AA, and rs7536540 CC/CG + rs2227513 AG.

$B$ : CDIA score was progressively increased in RA patients carrying the genotypes of rs7536540 GG + rs2227513 AA, rs7536540 GG + rs2227513 AG, rs7536540 CC/CG + rs2227513 AA, and rs7536540 CC/CG + rs2227513 AG. 


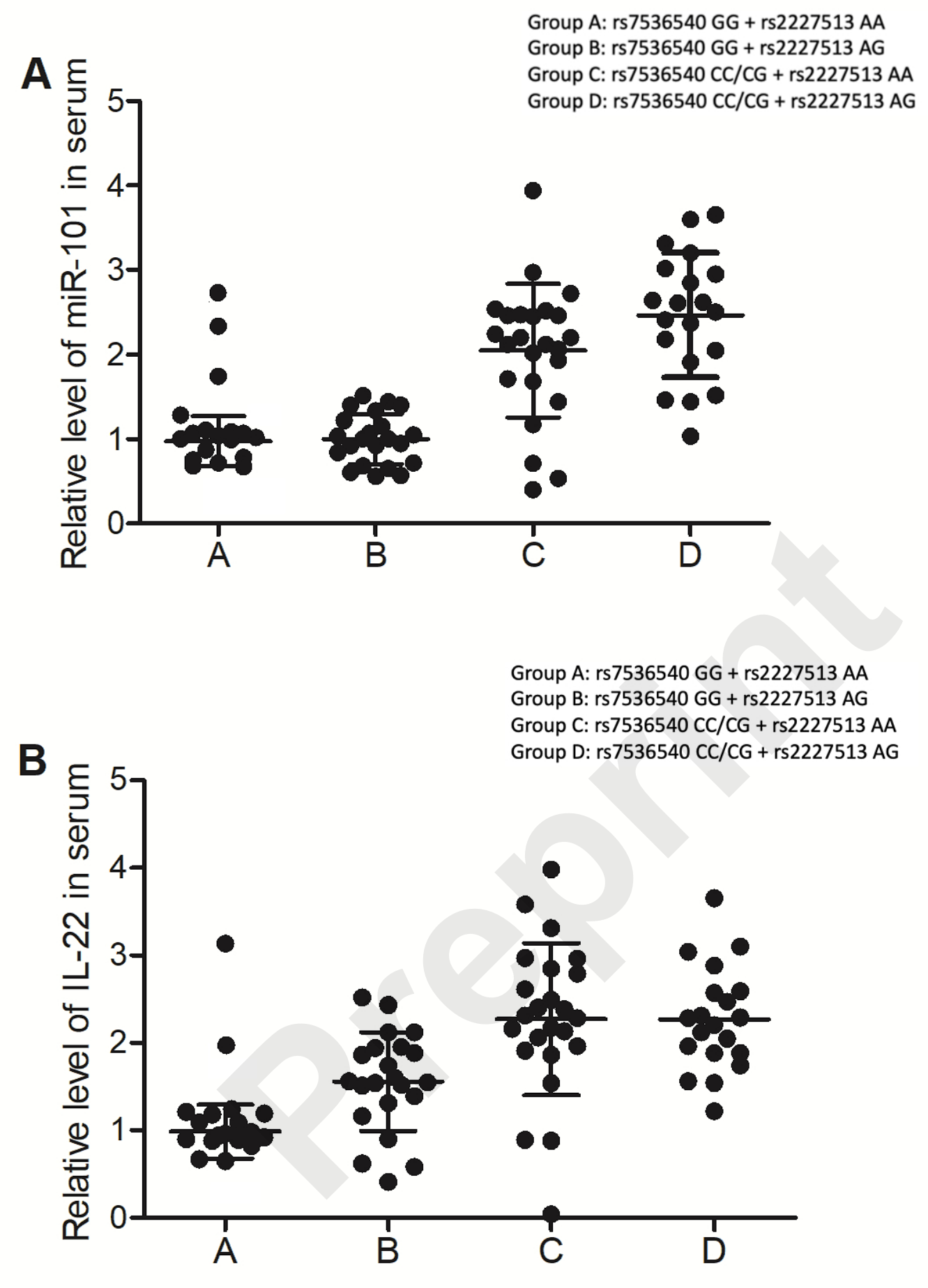

Fig. 2

CC/CG alleles at rs7536540 and AG alleles at rs2227513 were correlated with enhanced expression of miR-101 and IL-11 in the serum of RA patients.

A: The expression of miR-101 was significantly elevated in the serum of RA patients with CC/CG alleles at rs7536540.

$B$ : The expression of IL-22 was gradually enhanced in the serum of RA patients carrying the genotypes of rs7536540 GG + rs2227513 AA, rs7536540 GG + rs2227513 AG, rs7536540 $\mathrm{CC} / \mathrm{CG}+\mathrm{rs} 2227513 \mathrm{AA}$, and rs7536540 CC/CG + rs2227513 AG. 

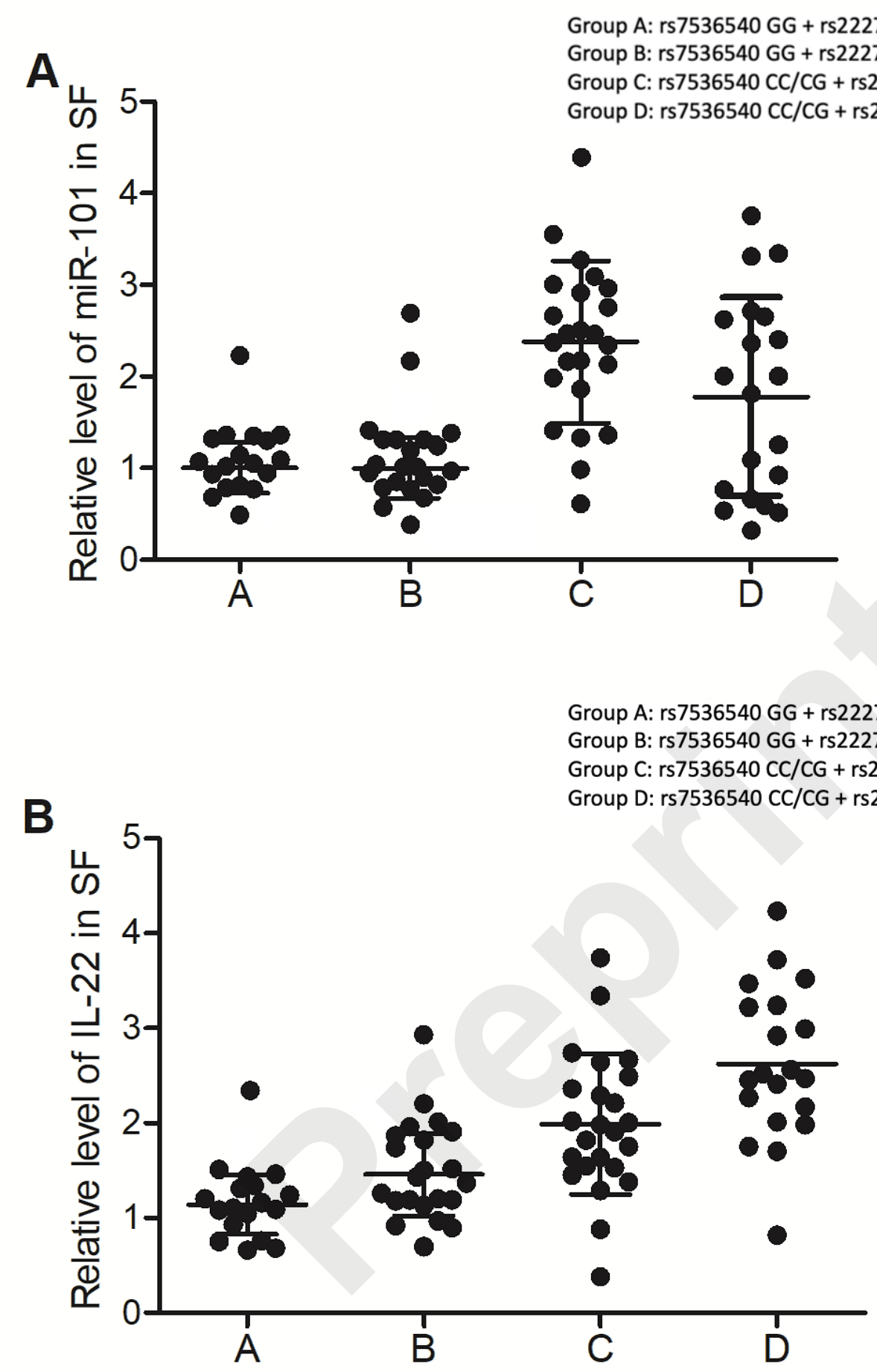

Fig. 3

CC/CG alleles at rs7536540 and AG alleles at rs2227513 were correlated with enhanced expression of miR-101 and IL-11 in the SF of RA patients.

A: The expression of miR-101 was significantly elevated in the SF of RA patients with CC/CG alleles at rs7536540.

B: The expression of IL-22 was gradually enhanced in the SF of RA patients carrying the genotypes of rs7536540 GG + rs2227513 AA, rs7536540 GG + rs2227513 AG, rs7536540 $\mathrm{CC} / \mathrm{CG}+\mathrm{rs} 2227513 \mathrm{AA}$, and rs7536540 CC/CG + rs2227513 AG. 


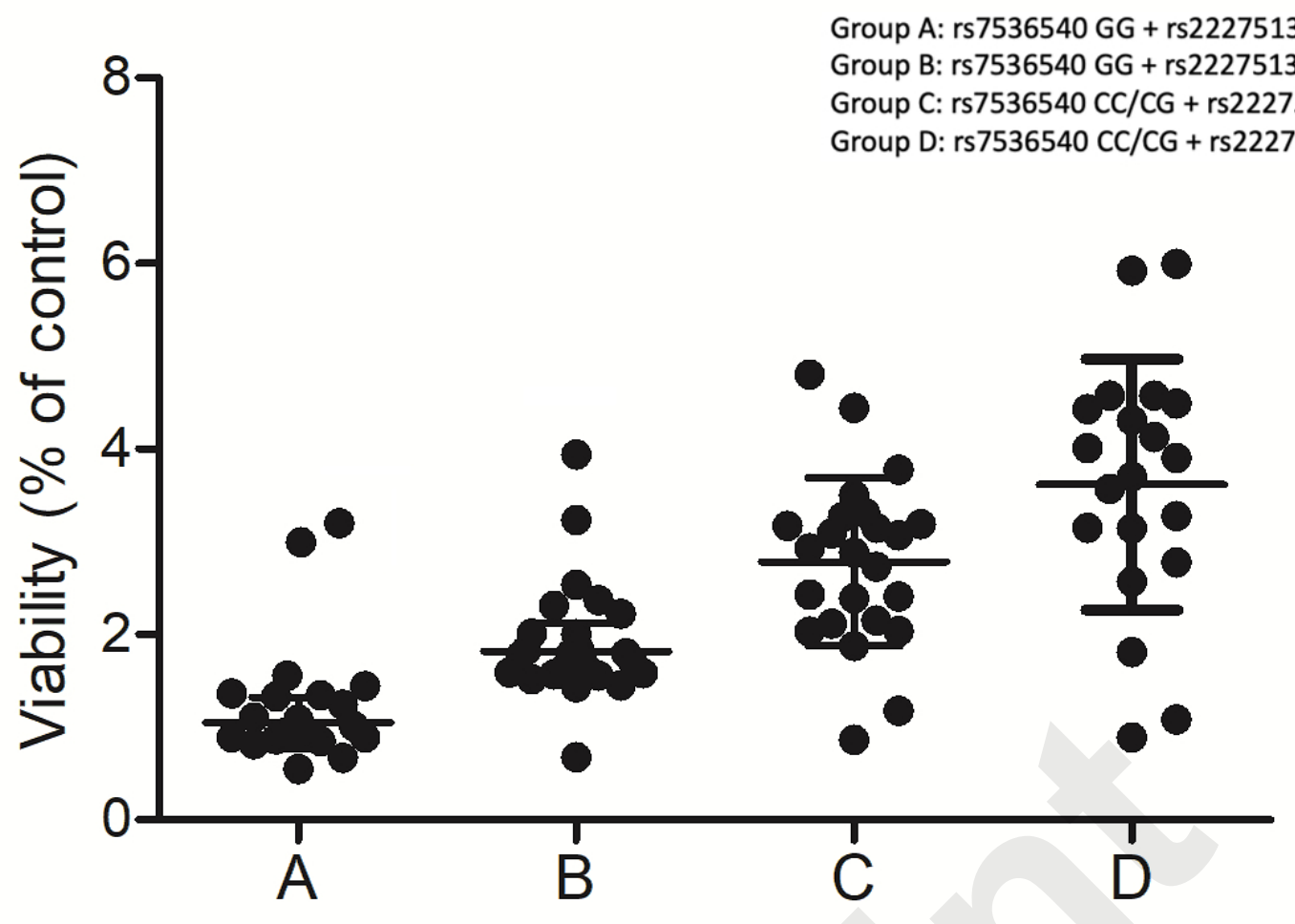

Fig. 4

The viability of FLS was gradually elevated when incubated with SF isolated from RA patients carrying the genotypes of rs 7536540 GG + rs2227513 AA, rs7536540 GG + rs2227513 AG, rs7536540 CC/CG + rs2227513 AA, and rs7536540 CC/CG + rs2227513 AG. 


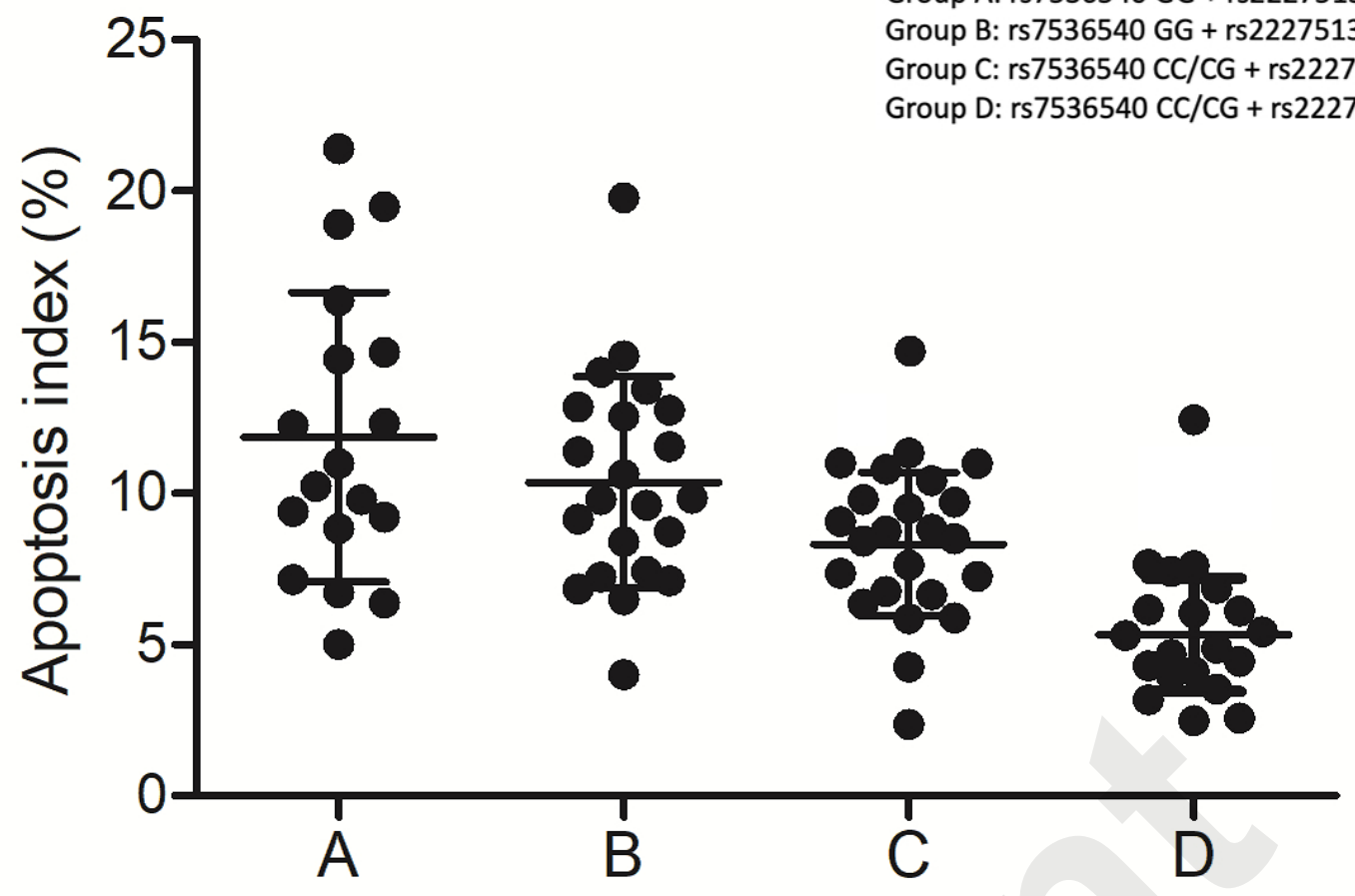

Fig. 5

The apoptosis of FLS was progressively decreased when incubated with SF isolated from RA patients carrying the genotypes of rs7536540 GG + rs2227513 AA, rs7536540 GG + rs2227513 AG, rs7536540 CC/CG + rs2227513 AA, and rs7536540 CC/CG + rs2227513 AG. 


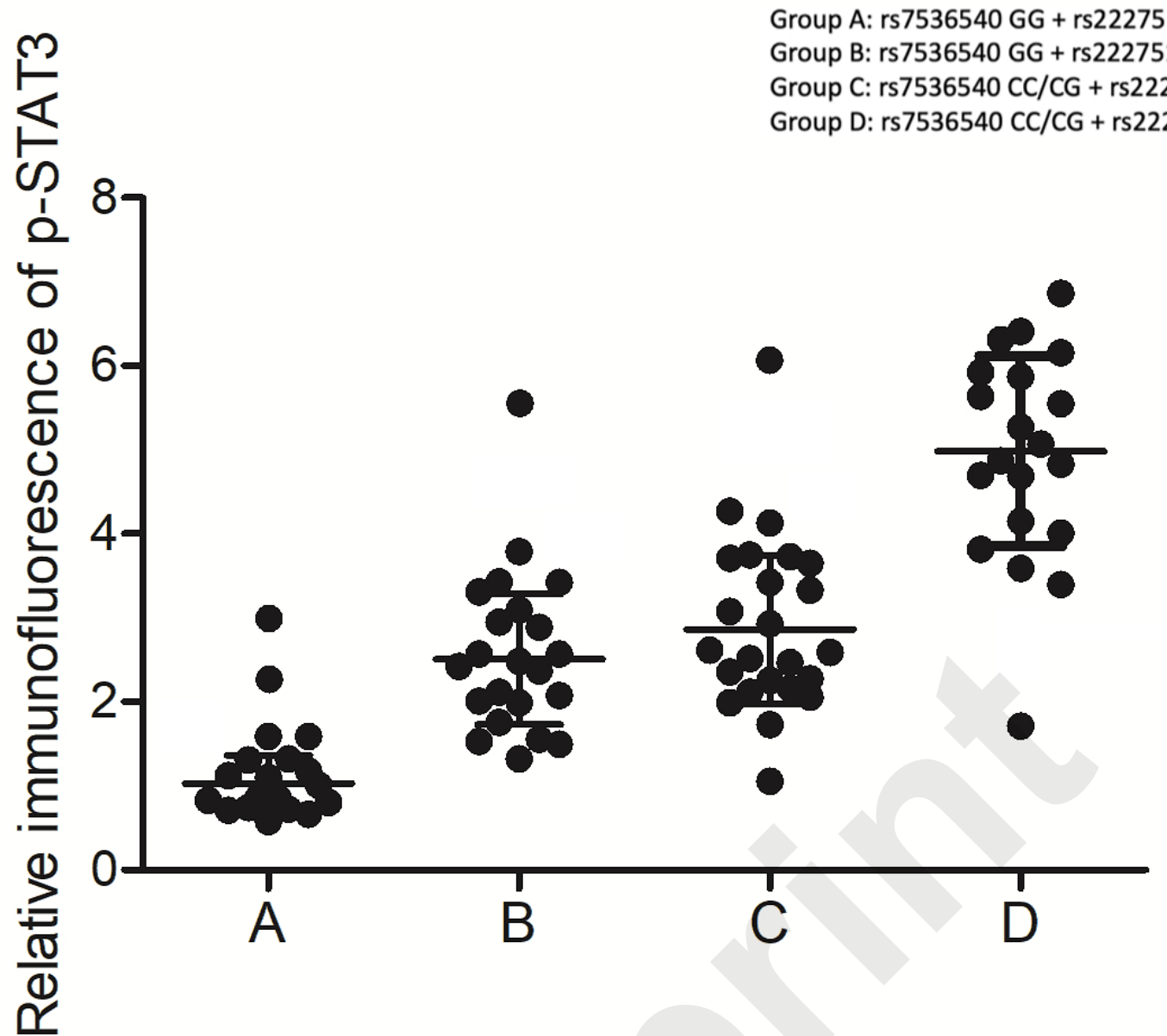

Fig. 6

The expression of p-STAT3 was gradually elevated when incubated with SF isolated from RA patients carrying the genotypes of rs7536540 GG + rs2227513 AA, rs7536540 GG + rs2227513 AG, rs7536540 CC/CG + rs2227513 AA, and rs7536540 CC/CG + rs2227513 AG. 
B

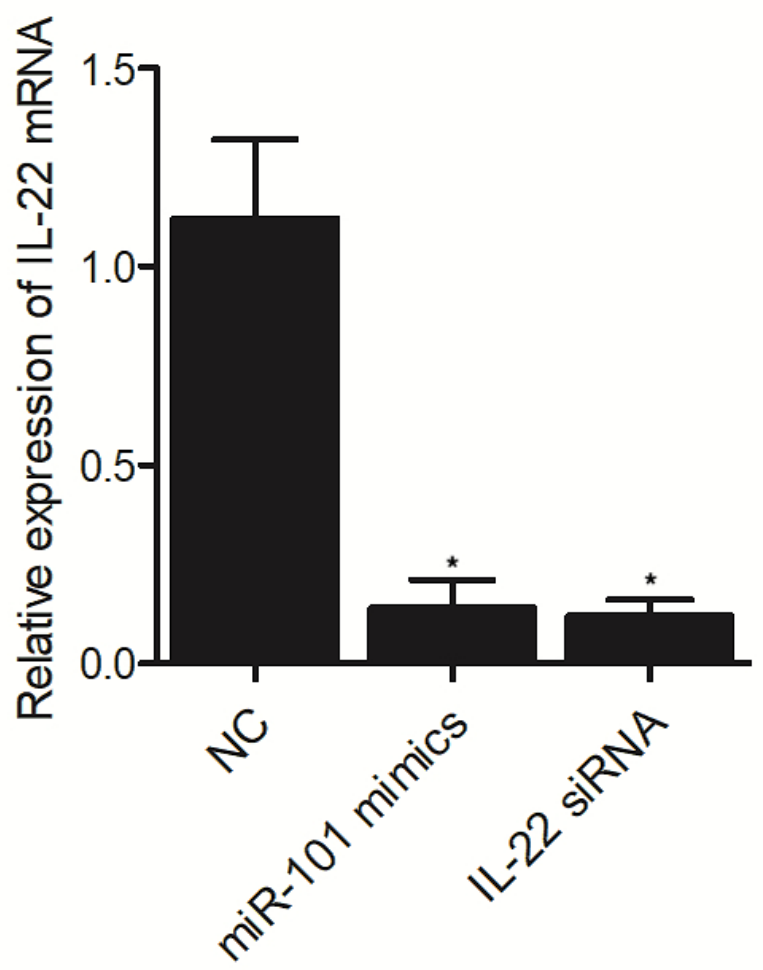

\section{$\beta$-actin}

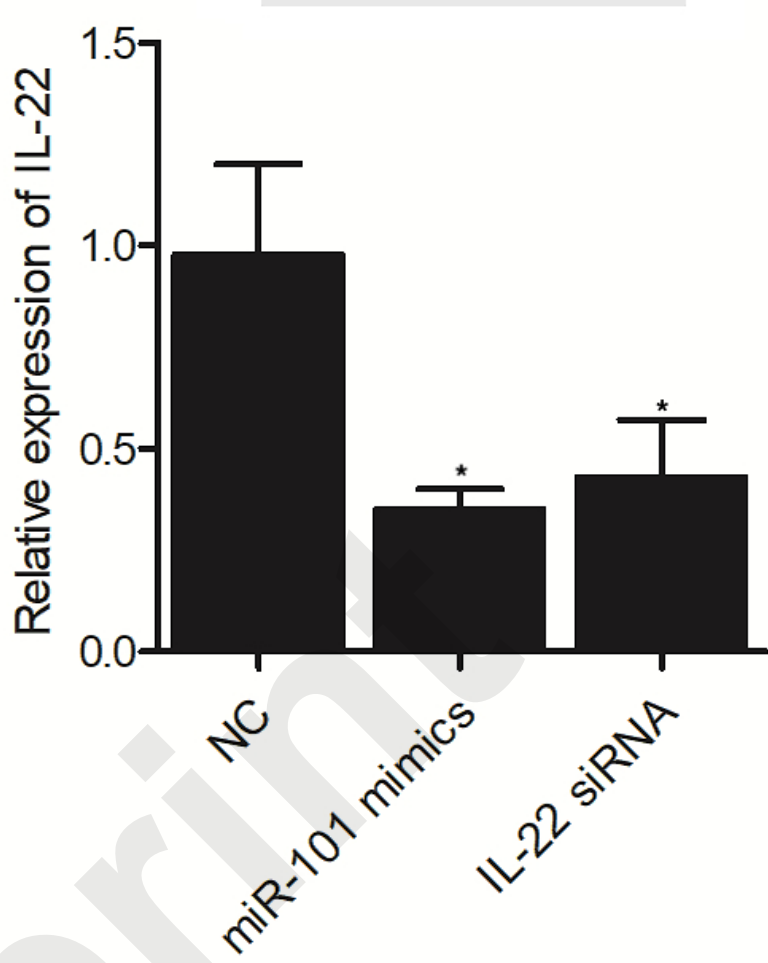

Fig. 7

The expression of IL-22 in THP-1 cells was inhibited by miR-101 mimics and IL-22 siRNA. A: The expression of IL-22 mRNA was remarkably suppressed by miR-101 mimics and IL-22 siRNA ( ${ }^{*} P$ value $<0.05$, vs. NC group).

$\mathrm{B}$ : The expression of IL-22 protein was remarkably suppressed by miR-101 mimics and IL-22 siRNA ( ${ }^{*} \mathrm{P}$ value $<0.05$, vs. NC group). 
A

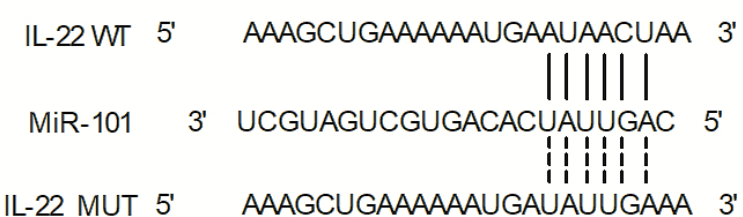

B

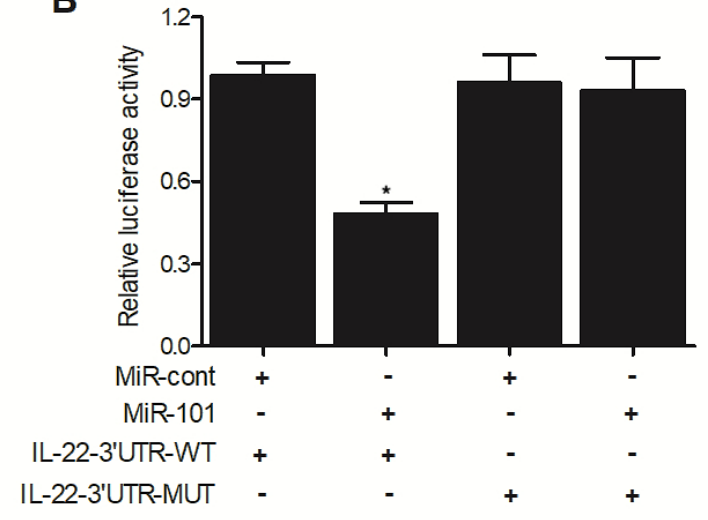

Fig. 8

MiR-101 inhibited the expression of IL-22 through binding to its 3' UTR.

A: Sequence analysis indicated a potential binding site of miR-101 on IL-22.

B: The luciferase activity of wild type IL-22 was effectively repressed by miR-101 ( ${ }^{*} \mathrm{P}$ value $<0.05$, vs. control group). 